\title{
XVI.
}

\section{Ueber die Verbindungen der Borsäure und des Wassers mit dem Bleioxyd und dem Kupferoxyd.}

\author{
Von \\ Ti. Rose.
}

(A. d. Ber. d. Berl. Akad.)

Mit dem Bleioxyd verbindet sich die Borsäure und das Wasser in den mannigfaltigsten Verhältnissen; bei wenigen anderen Verbindungen kann der Einfluss des Wassers bei verschiedenen Temperaturen und bei verschiedenen Mlengen so nachgewiesen werden, wie bei diesen. In seiner Verwandtschaft zur Borsāure steht das Bleioxyd den alkalischen Erden weit nach, da diese sich mit der Bol'săure und dem Wasser vorzugsweise nur in einigen Verbāltnissen verbinden.

Wenn man die Losungen gleicher Atomgewicbte von salpetersaurem Bleioxyd und von neutralem Borax (Nä̈) in der Kälte mit einander vermischt, so erhält man nicht neutrales borsaures Bleioxyd, sondern eine Fälung von der Zusammensetzung $3(\dot{\mathrm{P}} b \overrightarrow{\mathrm{B}}+\dot{\mathrm{H}})+\dot{\mathrm{P}} \mathrm{b} \dot{\mathrm{H}}+\dot{\mathrm{H}}$, die aber durch Auswaschen mit kaltem Wasser noch mehr Borsäure verliert und sich dadurch in $(\dot{\mathbf{P}} \mathrm{b} \ddot{\mathbf{B}}+\dot{\mathbf{H}})+\dot{\mathbf{P}} \mathbf{b} \dot{\mathbf{H}}$ verwandelt. Werden die Lossungen beider Salze heiss mit einander in Berūhrung gebracht, so ist die unausgewaschene Fällung von der Zusammensetzung ( $\dot{\mathrm{P} b} \ddot{\mathbf{B}}+\dot{\mathbf{H}}$ ) $+\dot{\mathrm{P}} b \dot{\mathrm{H}}$, die mit. heissem Wasser ausgewaschene bingegen $3(\dot{\mathrm{P}} b \ddot{\mathrm{B}}$ $+\dot{\mathrm{I}})+5 \dot{\mathrm{P} b} \dot{\mathrm{H}}$.

Bei der Zersetzung von kalten concentrirten Lüsungen von salpetersaurem Bleioxyd und von zweifach-borsaurem Natron entsteht eine Fāllung von der Zusammensetzung $\dot{\mathrm{P}}_{\mathrm{b}} \dddot{\mathrm{B}}_{2} \dot{\mathrm{H}}+$ $8(\dot{\mathrm{P} b} \ddot{\mathrm{B}} \dot{\mathrm{H}})$, die also der des neutralen borsauren Bleioxyds sich sehr năhert. Durcl Auswaschen wird sie ganz neutral und ist dann $\dot{\mathrm{P}} b \ddot{\mathrm{B}} \dot{\mathrm{H}}$. Kalte sehr verdünnle Auflōsungen geben die Fāllung $5 \dot{\mathrm{P} b} \ddot{\mathrm{B}} \ddot{\mathrm{H}}+4 \dot{\mathrm{P} b} \dot{\mathrm{H}}$. 
Durch die Zersetzung von heissen concentrirten Losungen der Salze erbält man die Fällung $5 \dot{\mathrm{P}} \mathrm{b} \ddot{\mathbf{B}} \dot{\mathbf{H}}+\dot{\mathrm{Pb}} \dot{\mathbf{H}}$, die durch das Auswaschen nit heissem Wasser sich in die Verbindung $3 \dot{\mathrm{P}} \mathrm{b} \ddot{\mathrm{B}} \dot{\mathrm{H}}+\dot{\mathrm{P}} \mathrm{b} \dot{\mathrm{H}}$ verwandelt. Durch beisse sehr verdünte Lósungen entstebt der Niederscblag $\dot{\mathrm{Pb}} \ddot{\mathrm{B}} \dot{\mathrm{H}}+\dot{\mathrm{Pb}} \dot{\mathrm{H}}$, der von allen untersuchten Verbindungen der Borsäure mit dem Bleioxyd, die durch ${ }^{-}$geworhnlichen Borax erzeugt worden sind, am meisten Bleioxyd gegen Borsāure enthält.

Noch leichter als bei den Verbindungen der Borsäure mit dem Bleioxyd kann bei denen dieser Sảure mit dem Kupferoxyd das Wasser einen Theil der Borsāure austreiben. Es ist sogar mőglich, vermittelst einer grossen Menge von Wasser, wenn die Einwirkung desselben durch eine erhőhte Temperatur unterstüıt wird, endlich alle Borsãure vom Kupferoxyd zu trennen.

Werden die concentrirten Losungen gleicher Atomgerrichte von schwefelsaurem Kupferoxyd und von neutralem Borax kalt mit einander vermischt, so erhălt man eine Fällung von $5(\dot{C} u \bar{B}$ $+\dot{\mathbf{H}})+4 \dot{\mathrm{C} u} \dot{\mathrm{H}}$, aber gemengt mit schwefelsaurem Natron und unlüslichem basisch-schwelelsaurem Kupferoxyd. Nach dem Auswaschen mit kaltem Wasser ist dic Verbindung $2(\dot{\mathrm{Cu}} \ddot{\mathrm{B}}+\dot{\mathbf{H}})+$ $4 \dot{\mathrm{C}} \dot{\mathrm{H}}$ gemengt mit etwas basisch-schwefelsaurem Kupferoxyd. Heisse concentrirte Lossungen beider Salze geben einen Niederschlag von der Zusammensetzung $5(\dot{C} u \ddot{B}+\dot{I})+6 \dot{C} u \dot{H}$, gemengt mit schwefelsaurem Natron und basisch-schwefelsaurem Kupferoxyd. Wird derselbe aber mit heissem Wasser ausgewaschen, so hat er wesentlich die Zusammensetzung von $4 \dot{C} u \ddot{B} \dot{H}$ $+7 \dot{\mathrm{C} u \dot{\mathrm{H}}}$, gemengt oder verbunden mit $2 \dot{\mathrm{C}} \mathrm{u}_{3} \overline{\mathrm{S}}$.

Das zweifache borsaure Natron giebt mit schwefelsaurem Kupferóxyd in concentrirten Lơsungen in der Kălte die Verbindung von $20 \dot{C} u \ddot{B} \dot{H}$ mit $13 \dot{C} u \dot{\Psi}$, getnengt mit $\dot{C} u_{3} \ddot{S}$ und $2 \dot{N} a \vec{S}$, die aber durch das Auswaschen mit kaltem Wasser Borsăure verliert und $\dot{\mathrm{C}} \overrightarrow{\mathrm{B}} \dot{\mathrm{H}}+\dot{\mathrm{C}} \mathrm{i} \dot{H}$, gemengt mit etwas basisch-schwefelsaurem Kupferoxyd, wird: Heisse concentrirte Auflosungen

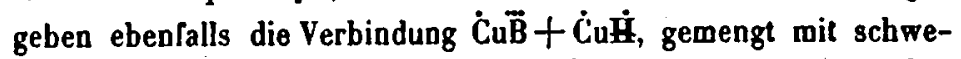
felsaurem Natron und basisch-schwefelsaurem Kupferoxyd, aber 
schon durchs Auswaschen mit kaltem Wasser verliert sie Borsãure und wird $\dot{C} u \ddot{B}+3 \dot{C} u \dot{H}$, gemengt mit etwas $\dot{C}_{u_{3}} \ddot{\mathbf{S}}$ und noch weniger $\dot{\mathrm{N}}_{\mathrm{a}} \overrightarrow{\mathrm{S}}-$; nach dem Auswaschen aber mit heissem Wasser ist sie $4 \dot{C} u \ddot{B} \dot{H}+5 \dot{C} u \dot{H}$, gemengt mit $2 \dot{C} u_{3} \dot{S}$ und etwas schwefelsaurem Natron.

Kalte verdūnnte Lusungen der Salze geben eine Verbindung

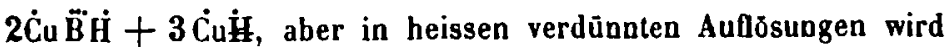
durch die grosse Menge Wasser der Fällung so viele Borsăure entzogen, dass sie nach dem Auswaschen die Zusammensetzung $\dot{\mathrm{C} u} \overrightarrow{\mathrm{B}} \dot{\mathrm{H}}+(10 \dot{\mathrm{C}} \mathrm{u}+8 \dot{\mathrm{\Psi}})$ hat. Wird bei verdünnten Lōsungen das Kochen noch einige Zeil hindurch fortgesetzt, so kann man endlich einen Niederscblag erhalten, aus welchem alle Borsäure ausgewaschen ist und der wesentlich aus $10 \dot{C} u+3 \dot{\#}$ besteht.

\title{
XVII.
}

\section{Ueber die Verbindungen der Borsäure und des Wassers mit dem Kobaltoxyde, dem Nickeloxyde, dem Zinkoxyde und dem Cudmiumoxyde.}

\author{
Von \\ II. Hose.
}

(A. d. Ber. d. Berl. Akad.)

Es wurden von diesen borsauren Verbindungen nur die Niederschläge der Untersuchung unterworfen, welche vermittelst der Auflosung des gewzhnlichien Borax in den Auflosungen der schwefelsauren Salze der genannten Oxyde erbalten worden waren.

Das borsaure Kobaltoxyd, welches durch Fällung von Ldsungen gleicher Atomgewiclite von Borax und von schwefelsaurem Kobaltoxyd in der Kálle entsteht, ist in Wasser niebt vollkommen unlosslich, das Wascluwasser ist daher rủthlich gefărbı̣, und trübt sich durcbs Zusetzen von Losungen sowohl von Borax, als auch von schwefelsaurem Kobaltoxyd. Es enthält nach dem Aus- 